\title{
Demencia rápidamente progresiva, un reto diagnóstico. A propósito de dos casos clínicos de demencia secundaria a enfermedad de Creutzfeldt-Jakob en una misma familia en Ecuador
}

Vanesa Berrú ${ }^{\text {, Brenda Pillajo-Sánchez }}{ }^{2}$

'Servicio de Geriatría y Cuidados Paliativos. Hospital San Juan de Dios. Quito. Ecuador

${ }^{2}$ Geriatría. Pontificia Universidad Católica del Ecuador. Quito. Ecuador

Recibido:01/09/2018

Aceptado: 16/11/2018

En línea: 31/12/2018

Citar como: Berrú V, Pillajo-Sánchez B. Demencia rápidamente progresiva, un reto diagnóstico. A propósito de dos casos clínicos de demencia secundaria a enfermedad de Creutzfeldt-Jakob en una misma familia en Ecuador. Rev Esp Casos Clin Med Intern (RECCMI). 2018 (Dic); 3(3): 136-139. doi: 10.32818/reccmi.a3n3a12.

Autor para correspondencia: Vanesa Berrú. vanesa.berru@sanjuandedios.ec

\section{Palabras clave \\ $\triangleright$ Encefalopatía espongiforme \\ $\triangleright$ Enfermedad de Creutzfeldt- Jakob \\ $\triangleright$ Enfermedad por priones}

\section{Keywords \\ $\triangleright$ Spongiform encephalopathy \\ $\triangleright$ Creutzfeldt-Jakob disease \\ $\triangleright$ Prion disease}

\begin{abstract}
Resumen
Según la Organización Mundial de la Salud, la demencia es el deterioro crónico, global y habitualmente irreversible de la capacidad cognitiva. Existen varios tipos de demencia, entre ellas las rápidamente progresivas, que llevan a la muerte. Se presenta a continuación una serie de casos en una misma familia de Ecuador con demencia probablemente secundaria a enfermedad de Creutzfeldt-Jakob variante familiar. Esta entidad se caracteriza por deterioro cognitivo rápidamente progresivo y mioclonías. Con la revisión de estos casos, se pretende concienciar sobre la importancia de un estudio rápido y exhaustivo de pacientes que presenten deterioro cognitivo súbito y progresivo.

Abstract
According to the World Health Organization, dementia is the chronic, global and generally irreversible deteriora-
tion of cognition; there are several types of dementia, including those of subacute evolution, which are rare, known
as rapidly progressive, leading to the death of the patient. A series of cases of dementia secondary to familial va-
riant Creutzfeldt-Jakob disease in the same family in Ecuador is below. This disease is characterized by rapidly
progressive cognitive deterioration and myoclonus. We intend to raise awareness about the importance of a rapid
and exhaustive study of patients with sudden and progressive cognitive deterioration with the presentation of
clinical cases.
\end{abstract}

\section{Puntos destacados}

$\triangleright$ La epidemiología de la enfermedad de Creutzfeldt-Jakob familiar en el Ecuador, un país latinoamericano, no había sido reportada hasta ahora, siendo los dos primeros casos que se describen, y esto constituye una llamada de atención a las autoridades sanitarias del país para que la vigilancia epidemiológica de este tipo de demencia sea de particular interés.

$\triangleright$ Es necesario incluir el estudio genético en el protocolo de estudio de demencia rápidamente progresiva para evitar subregistros de las otras variantes.

\section{Introducción}

La enfermedad de Creutzfeldt-Jakob (ECJ) es la más común de las enfermedades priónicas del ser humano'. Los priones son moléculas compuestas por material proteico que pueden propagarse en un mismo huésped o transmitirse a otros en periodos prolongados de tiempo ${ }^{2}$. Existen formas de tipo esporádico, familiar y yatrogénico. La forma esporádica es la más común y constituye el $84 \%$ de los casos²; el 5-15\% son de tipo familiar y se transmiten de forma autosómica dominante por el gen PRNP2. Afecta por igual a hombres y mujeres, y los síntomas característicos son deterioro cognitivo y trastornos del movimiento, que se manifiestan en promedio a los 65 años de edad. La incidencia mundial es de 0.5-1 caso por millón de personas al año ${ }^{3}$. El diagnóstico, de acuerdo con los criterios de la Organización Mundial de la Salud (OMS) (Tabla 1), se estratifica en definitivo (inmunohistoquímico o neuropatológico), posible o probable. Estos últimos se apoyan en la determinación de proteínas (tau y 14-3-3) en líquido cefalorraquídeo; en hallazgos característicos en el electroencefalograma (complejos periódicos bifásicos o trifásicos, a intervalos de uno a varios segundos); en la aparición en la resonancia magnética cerebral del "signo del pulvinar" (imagen hiperintensa en pulvinar simétrica bilateral relacionada con la intensidad del resto de la sustancia gris en los núcleos basales y cortical). 
Berrú V, Pillajo-Sánchez B. Demencia rápidamente progresiva, un reto diagnóstico. A propósito de dos casos clínicos de demencia secundaria a enfermedad de Creutzfeldt-Jakob en una misma familia en Ecuador

\begin{tabular}{|c|c|}
\hline Diagnóstico & Características \\
\hline Definitivo & $\begin{array}{l}\text { Diagnóstico estándar, } \\
\text { técnicas de neuropatología/inmunohistoquímica }\end{array}$ \\
\hline Probable & $\begin{array}{l}\text { Demencia progresiva y dos de las siguientes } \\
\text { características clínicas: } \\
\text { - Mioclonías } \\
\text { - Disfunción visual o cerebelar } \\
\text { - Síntomas piramidales y extrapiramidales } \\
\text { - Mutismo acinético } \\
\quad \text { y } \\
\text { Electroencefalograma positivo } \\
\quad 0 \\
\text { Proteína 14-3-3 positivo }\end{array}$ \\
\hline Posible & $\begin{array}{l}\text { Demencia progresiva y dos de las siguientes } \\
\text { características clínicas: } \\
\text { - Mioclonías } \\
\text { - Disfunción visual o cerebelar } \\
\text { - Síntomas piramidales y extrapiramidales } \\
\text { - Mutismo acinético } \\
\quad \text { y } \\
\text { Duración menor de } 2 \text { años }\end{array}$ \\
\hline
\end{tabular}

Tabla 1. Criterios para la enfermedad de Creutzfeldt-Jakob (OMS, 1998)

No existe un tratamiento definitivo y la evolución de la enfermedad es la muerte pocos meses después del diagnóstico. A continuación, presentamos dos casos en una misma familia de Ecuador.

\section{Caso clínico 1}

Varón de 57 años, ecuatoriano, casado, antecedente de lesión de tipo papilomatoso (confirmado con biopsia) en pulgar izquierdo, no hay historia de consumo de fármacos. Antecedente familiar en su madre de ECJ, según consta en certificado de defunción (fallecida a los 52 años). El cuadro clínico es de 6 meses de evolución caracterizado por ataxia progresiva, que imposibilita la deambulación, disartria, alucinaciones visuales y auditivas con ideas de daño y perjuicio. En la exploración neurológica se destaca desorientación en tiempo, espacio y persona con lenguaje espontáneo, lentificado, con adecuada comprensión y denominación. Obedece comandos verbales simples y complejos. Fuerza muscular conservada, sensibilidad superficial y profunda conservada, normorreflexia global, maniobra de Stewart-Holmes positiva, presencia de disdiadococinesia, dismetría y marcha atáxica. Mini-Mental Test: 22/30 (deterioro cognitivo leve). Se plantean como diagnósticos diferenciales: encefalopatía por priones (enfermedad de Creutzfeldt-Jakob), demencia con cuerpos de Lewy, encefalopatías autoinmunes, encefalopatías infecciosas, linfoma cerebral, causas metabólicas y pseudodemencia asociada a depresión.

\section{Pruebas complementarias}

Los estudios básicos de laboratorio fueron normales. Bajo la sospecha de síndrome paraneoplási$\mathrm{CO}$, se realizaron marcadores tumorales (anti-Hu y anti-Yo), que resultaron negativos. El rastreo tomográfico y el análisis del líquido cefalorraquídeo (glucosa, proteínas y celularidad) fueron normales.
El electroencefalograma muestra trazo anormal por la presencia de actividad paroxística frontotemporal bilateral de predominio derecho aislada (Figura 1).

La resonancia magnética cerebral evidencia restricción de señal en corteza parietotemporal bilateral, hiperintensidad en núcleo caudado y putamen (Figura 2).

La identificación de proteína 14-3-3 en líquido cefalorraquídeo resultó positiva; igual que la proteína tau: $1788 \mathrm{pg} / \mathrm{ml}$ (punto de corte: $1150 \mathrm{pg} / \mathrm{ml}$ ).

\section{Evolución}

El cuadro clínico evoluciona con ataxia, temblor y deterioro cognitivo rápidamente progresivo. Ante la sospecha de encefalitis paraneoplásica, recibió cinco dosis de metilprednisolona, sin respuesta al tratamiento.

El paciente es ingresado, de acuerdo con los criterios de la OMS, con diagnóstico probable de ECJ. No se realiza biopsia cerebral ante la negativa de familiares.

Seis meses posteriores al inicio de los síntomas, el paciente desarrolla apraxia de la deglución, ante lo cual reingresa para realización de gastrostomía de alimentación, sin embargo fallece por neumonía nosocomial.

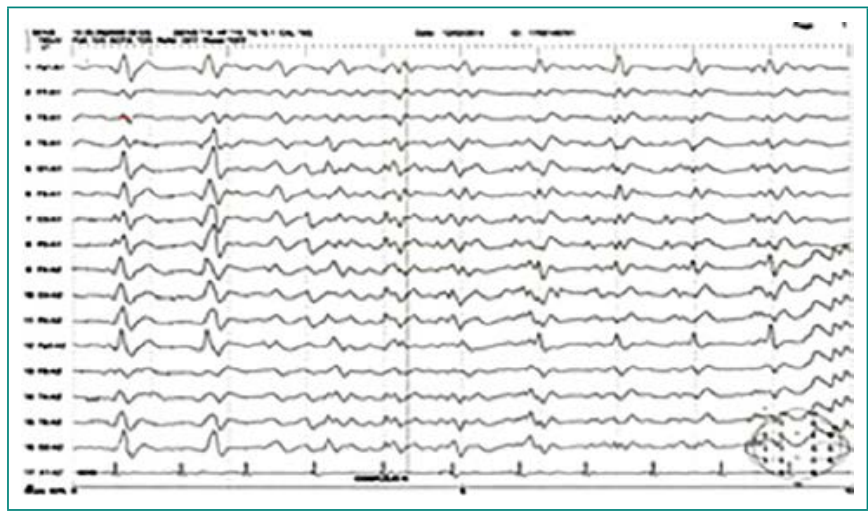

Figura 1. Complejos periódicos de ondas agudas a intervalos de 1 a 2 segundos

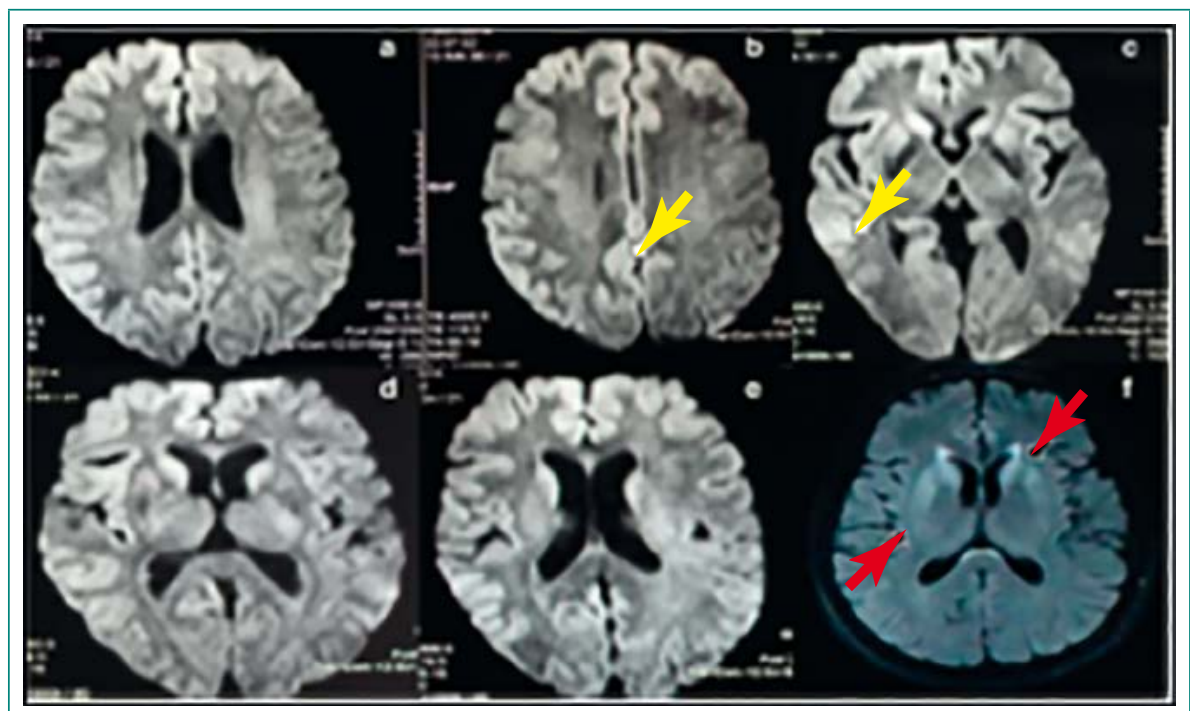

Figura 2. Restricción de señal en corteza parietotemporal bilateral (flechas amarillas), hiperintensidad en núcleo caudado y putamen (flechas rojas) 


\section{Caso clínico 2}

Mujer de 65 años, ecuatoriana, viuda, autónoma e independiente, antecedente familiar de hermano fallecido a los 57 años con probable ECJ, descrito previamente, además de otro hermano en estudio de deterioro cognitivo rápidamente progresivo mientras se desarrolla esta revisión, y madre fallecida (52 años) por ECJ (según certificado de defunción). La paciente no tenía antecedente de enfermedades previas, ni consumo de fármacos. Presentó en los últimos 3 meses ideas delirantes de tipo daño y perjucio, alucinaciones visuales, auditivas y soliloquios, acompañado de deterioro cognitivo progresivo, agresividad, afectación en actividades básicas e instrumentales de la vida diaria y alteración del equilibrio (caídas frecuentes). Fue catalogada y tratada como trastorno de ideas delirantes más psicosis, y recibió dosis bajas de antipsicóticos atípicos. Sin embargo, el deterioro neurológico progresa con desorientación, bradipsiquia, alteración de funciones mentales superiores y mioclonías en miembro superior derecho. No se evidencian signos de enfermedad en resto de aparatos y sistemas. Mini-Mental Test: 2/30.

\section{Pruebas complementarias}

Los estudios de laboratorio (como hemograma, química sanguínea, perfil metabólico, hepático, tiroideo, electrólitos) fueron normales. El estudio de líquido cefalorraquídeo (glucosa-proteínas y celularidad) fue normal.

El electroencefalograma reportó anormalidad por actividad periódica continua generalizada y descargas epileptiformes mioclónicas frecuentes en un fondo lento, todo ello indicativo de encefalopatía severa.

La tomografía cerebral muestra adecuada densidad del parénquima cerebral, no lesiones intraaxiales ni extraaxiales, estructuras de línea media no desplazadas, sistema cisternoventricular de amplitud normal, adecuada diferenciación sustancia blanca y gris (Figura 3). El estudio de imagen cerebral por resonancia magnética mostró incremento de la señal en núcleos caudado y putamen.
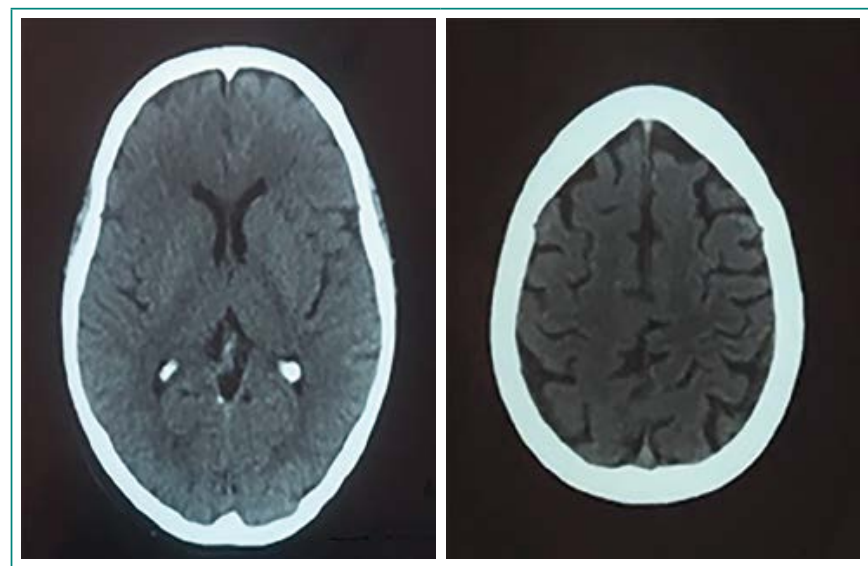

Figura 3. Tomografía axial computarizada cerebral (corte axial): atrofia cortical y subcortical

Dados los antecedentes previos en la familia de la paciente, se solicitó estudio genético (Laboratorio INVITAE San Francisco - California) para investigación del gen PRNP, y el resultado fue positivo variante heterocigoto c.598G>A (p.Glu200Lys)

\section{Evolución}

Durante su evolución, se destaca la presencia de apraxia deglutoria, afasia, signos extrapiramidales e hiperreflexia osteotendinosa global y episodios frecuentes de agitación psicomotriz. Finalmente, la paciente fallece posterior a los
3 meses de evolución de la sintomatología. Con estos antecedentes, se cataloga como deterioro cognitivo rápidamente progresivo secundario a probable ECJ variante familiar, de acuerdo con los criterios de la OMS. No se solicitó necropsia para confirmar el diagnóstico.

\section{Discusión}

El estudio de la demencia rápidamente progresiva incluye un trabajo diagnóstico exhaustivo. Las causas más importantes incluyen las encefalopatías por priones, particularmente la ECJ; enfermedades neurodegenerativas, como la demencia con cuerpos de Lewy; encefalopatías autoinmunes, como la encefalitis límbica (paraneoplásica o no paraneoplásica) y las encefalopatías que responden a corticosteroides; vasculitis; infecciones como la enfermedad de Whipple; neoplasias, particularmente el linfoma intravascular; causas toxicometabólicas y la pseudodemencia asociada a depresión ${ }^{4}$.

La ECJ es una encefalopatía espongiforme rápidamente progresiva y fatal. Se clasifica en esporádica, familiar o adquirida. La forma familiar de la ECJ debuta, por lo general, más tempranamente, tiene un curso más rápido y mayor postración. Esta variante, junto con otras formas familiares de enfermedades priónicas, como el insomnio fatal familiar y la enfermedad de Gerstmann-Straussler-Scheinker, representa sólo el 10\% de los casos $^{5}$. Clínicamente, es muy parecida a la variante esporádica, es decir, se presenta con una combinación de demencia progresiva, déficits corticales focales como afasia y trastornos visuoespaciales, manifestaciones extrapiramidales y/o cerebelosas, mioclonías y trastornos de conducta o psiquiátricos ${ }^{6}$. Se manifiesta usualmente entre los 50-70 años y tiene una sobrevida promedio de 5 meses. Aproximadamente el $85 \%$ de los pacientes fallece dentro del primer año del comienzo de los síntomas. Los casos presentados se definen como caso probable de ECJ, de acuerdo con los criterios de la OMS, pues no fue posible la confirmación histopatológica (gold estándar).

En condiciones normales, el cerebro presenta proteína priónica (PrPc). La forma insoluble (anormal) de esta proteína priónica (PrPsc) es la que se acumula formando agregados en la ECJ. Existen más de 50 mutaciones del gen PRNP descritas, las mismas se transmiten de forma autosómica dominante con alta penetrancia e incidencia que incrementa con la edad. La mutación frecuentemente registrada en todo el mundo es E200K, que implica una sustitución de glutamato (E) por lisina (K) en el codón 200. En concreto, las mutaciones E200K, D178N, P102L, V210l y la inserción de 5-octopéptidos suponen más del 95\% del total, siendo el resto excepcionales ${ }^{7}$. La mutación E200K se ha descrito prácticamente en toda Europa, Chile, Japón y Estados Unidos, sin ser Ecuador la excepción. Se presentan dos probables casos en una misma familia, uno de ellos apoyado por el hallazgo de la mutación E200K del gen PRN. Chile, un país vecino, reporta una alta incidencia a nivel mundial, llegando al 22\% en la variante $\mathrm{fECJ}$, con una edad promedio de muerte de 55 años $^{8}$.

\section{Bibliografía}

1. Mahboob HB, Kaokaf KH, Gonda JM, Creutzfeldt-Jakob disease presenting as expressive aphasia and nonconvulsive status epilepticus, case reports in critical care. [Internet]. 2018; vol. 2018: article ID 5053175. Disponible en: https://www.hindawi.com/journals/cricc/2018/5053175/

2. DeArmond SJ, Prusiner SB. Etiology and pathogenesis of prion diseases. The American Journal of Pathology. [Internet]. 1995; 146(4): 785-811. Disponible en: https://www.ncbi.n/m.nih.gov/pmc/articles/PMC1869256/

3. Seed C, Hewitt P, Dodd R, Houston F, et al. Creutzfeldt-Jakob disease and blood transfusion safety. Vox Sanguinis. [Internet]. 2018. Disponible en: https://www.pptaglobal.org/images/publications/2018/CJD_Seed_et_al2018-Vox_Sanguinis_Cervenakova.pdf 
4. Blumenkron D, Guerrero P. Enfermedad de Creutzfeldt-Jakob. Med Int Mex. [Internet]. 2007; 23(1): 34-46. Disponible en: http://cmim.org/boletin/ pdf2007/MedlntContenido 01_08.pdf

5. Geschwind MD, Shu H, Haman A, Sejvar JJ, Miller BL. Rapidly progressive dementia. Ann Neurol. [Internet]. 2008; 64(1): 97-108. Disponible en: https:// www.ncbi.n/m.nih.gov/pmc/articles/PMC2647859/

6. Kretzschmar HA, Ironside JW, De Armond SJ, et al. Diagnostic criteria for sporadic Creutzfeldt-Jakob disease. Arch Neurol. [Internet]. 1996; 53(9): $913-$ 920. Disponible en: https://jamanetwork.com/journals/jamaneurology/ article-abstract/594202

7. González A, Montero Escribano P, Morlán Gracia L, Martínez-Menéndez B, Aladro Benito $Y$, et al. Enfermedad de Creutzfeldt-Jakob genética ful- minante asociada a mutación E200K y polimorfismo M129V. Neurol Arg. [Internet]. 2011; 3(4): 210-213. Disponible en: http://www.elsevier.es/ es-revista-neurologia-argentina-301-articulo-enfermedad-creutzfeldtjakob-genetica-fulminante-asociada S1853002811000899\#elsevierltemB ibliografias

8. Espinoza C, Henríquez S. Epidemiología de la enfermedad de Creutzfeldt Jakob en Chile entre los años 2001 a 2007. Estudio de tasas regionales de morbimortalidad. Tesis. Universidad de Chile Facultad de Medicina Escuela de Kinesiologia. [Internet]. 2011. Disponible en: http://repositorio.uchile.cl/bitstream/handle/2250/117373/Tesis\%20Final\% 20CJ. pdf? sequence $=1$ 